International Journal of Pure and Applied Mathematics

Volume 99 No. 4 2015, 399-410

ISSN: 1311-8080 (printed version); ISSN: 1314-3395 (on-line version)

url: http://www.ijpam.eu

doi: http://dx.doi.org/10.12732/ijpam.v99i4.2

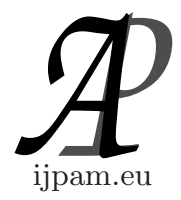

\title{
BUOYANCY EFFECTS OF THERMAL AND MASS DIFFUSION ON MHD NATURAL CONVECTION PAST FINITE VERTICAL, POROUS FLAT PLATE
}

\author{
Kinyanjui A. Muthondu ${ }^{1}$, Jeconia O. Abonyo ${ }^{2}$, \\ Muondwe Samuel $^{3 ~}$, Jacob Kirimi ${ }^{4}$, C. Isaak ${ }^{4}$ \\ ${ }^{1}$ Technical University of Kenya \\ ${ }^{2,3}$ Department of Pure and Applied Mathematics \\ Jomo Kenyatta University of Agriculture and Technology \\ P.O. Box 62000, Nairobi, KENYA \\ ${ }^{4}$ Chuka University \\ P.O. Box 60400, Chuka, KENYA
}

\begin{abstract}
The flow of an electrically conducting incompressible fluid due to buoyancy effects of thermal and mass diffusion past a finite vertical porous plate with constant suction was investigated in the presence of uniform transverse magnetic field. The problem has been solved for velocity, temperature and concentration profiles. The equations governing the flow are solved numerically using finite difference method for various values of Grashof parameter ranging from 0 to -1 . The results obtained are then presented using tables and graphs. It was noted that a decrease in Grashof parameter leads to an increase in primary, secondary, temperature and concentration profile.
\end{abstract}

AMS Subject Classification: 76E06

Key Words: buoyancy effects, mass diffusion, suction, magnetic field

Received: July 8, 2013

(C) 2015 Academic Publications, Ltd. url: www.acadpubl.eu

$\S_{\text {Correspondence author }}$ 


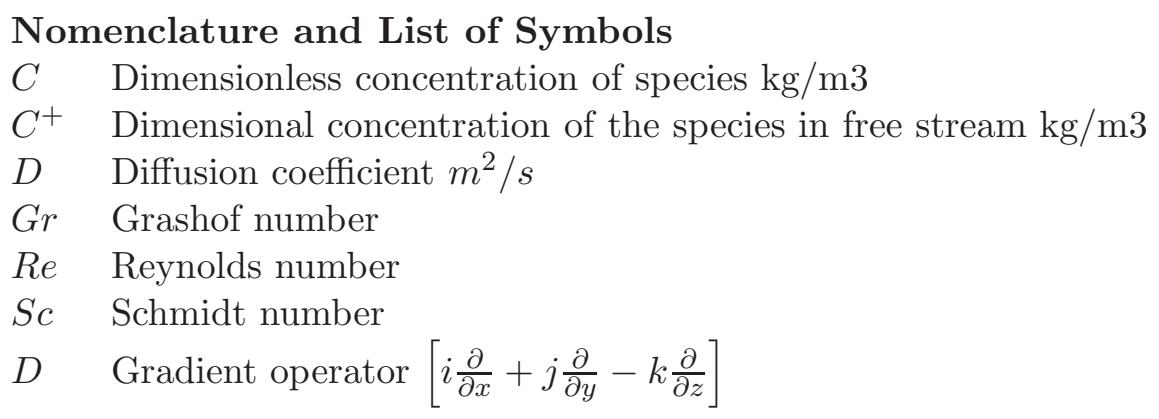

\section{Introduction}

The phenomenon of natural convection arises in fluids when temperature change causes density variation leading to buoyancy forces acting on the fluid particles. These flows which are driven by temperature differences abound in nature and have been studied extensively because of their application such as hot rolling and crystal growing glass fibre. In nature there exist flows that are caused not only by temperature differences but also by concentration differences. In industries many transport processes exists in which heat and mass transfer takes place simultaneously as a result of combined buoyancy effect of thermal diffusion and mass diffusion through chemical species. Free convection flows involving coupled heat and mass transfer occur frequently in nature. For this flows, the driving forces arises due to temperature and concentration variation of the fluid. For example in atmospheric flows, thermal convection resulting from heating of the earth by sunlight is affected by difference in water vapour concentration. A comprehensive literature on various aspects of natural convectional flow and its application coupled heat and mass transfer (Double diffusion) driven by buoyancy due to temperature and concentration variations in a saturated porous medium , has several important application in geothermal and geophysical engineering such as the migration of moisture through the air contained in fibrous insulation, the extraction of geothermal energy, underground disposal of nuclear waste, and the spreading of chemical contaminants through water saturated soil. The problem of combined heat and mass transfer over electrically conducting fluid in MHD free convection adjacent to a vertical surface are analysed by taking into account the effects of ohmic heating and viscous dissipation. Buoyancy forces resulting from thermal and mass diffusion is of considerable interest in nature and many industrial applications such as Geo-physics, solidification of binary alloys, drying process and chemical engineering. The analytical 1-D model compared favourable with the experiment 
results when modified to account for the hall parameter, the parameter was evaluated and correlation was developed. The hall parameter had a strong dependence disturbed the power output from the electrodes of high temperatures.

\section{Literature Review}

[3] presented free convection with mass transfer flow for a micro-polar fluid bounded by vertical infinite surface under the action of transverse magnetic field, the differential equations governing the problem were solved numerically by means of order Runge-kutta method. It was observed that the velocity decreases as prandtl number increases. Finally the concentration distribution is affected only by Schmidt number and decreases as this parameter increases. [4] analysed the effect of thermal radiation and viscous dissipation on MHD free convection flow over a semi-infinite vertical porous plate. The network simulation method was used to solve the boundary layer equation based on the finite difference formulation. It was found that an increase in viscous dissipation leads to an increase of both velocity and temperature profiles and a decrease in the velocity profiles. Finally an increase in the suction parameter leads to an increase in the local skin friction and Nusselt number. Also, when the Schmidt number was increased, the concentration level was decreased resulting in decreased fluid velocity. In addition, it was found that the skin friction coefficient increased due to increase in concentration buoyancy effect which it decreased due to increase in either heat absorption coefficient or Schmidt number.

[1] considered unsteady 2-D laminar, boundary layer flow over viscous, incompressible, electrically conducting and heat absorbing fluid along a semiinfinite vertical permeable moving plate in the presence of a uniform transverse magnetic field. Thermal and concentration buoyancy were discussed. The dimensionless governing equations for this investigation are solved analytically using two term harmonic and non-harmonic function, it was found that when the solution Grashof number increased, the concentration buoyancy effects were enhanced and thus, fluid velocity increased .however, the presence of heat absorption effect caused a reduction in the fluid temperature which resulted in a decrease in fluid velocity. Also, when the Schmidt number was increased, the concentration level was decreased resulting in decreased fluid velocity. In addition, it was found that the skin friction coefficient increased due to increase in concentration buoyancy effect which it decreased due to increase in either heat absorption coefficient or Schmidt number. 
[2] considered heat transfer in viscous flow along a plane wall with periodic suction and heat source. The effects of various parameters on heat transfers in a 3-D laminar boundary layer past a flat plate in the presence of a heat source when a sinusoidal transverse suction velocity was applied to the walls were studied.

[7] discussed their study on natural convection heat transfer in vertical open channel flows with discrete heating. Significant influences on the local heat transfer characteristics were noted, while only slight effects on the average characteristics were observed. [6] Investigated unsteady MHD flow through an inclined closed rectangular channel with upper and lower surfaces of varying permeability. In the same year [7] considered natural convection flow over a vertical frustum of a cone with constant heat flux. The numerical values of the velocity and temperature functions required to calculate the surface skin friction and heat transfer rates were discussed for various values of prandtl number.

Quadri and [1] considered simultaneous heat and mass transfer by natural convection for a vertical semi infinite plate embedded in a fluid saturated porous medium in the presence of wall suction and injection, heat generation or absorption effects, porous medium inertia and thermal dispersion effect. In general, the porous medium thermal dispersion effects increase the temperature of the fluid causing higher flow rate along the surface. However this seems not to be the case in their study as the peak values of the temperature and velocity profile were lowered as porous medium thermal dispersion parameter increases.

[5] investigated coupled heat and mass transfer by natural convection from a horizontal line course in a saturated porous medium. An analysis of boundary layer coupled heat and mass transfer from a line source in saturated porous medium was reported. The equations were numerically integrated for a range of Lewis number and buoyancy ratio, for aiding and opposing buoyancy effects.

The influence of viscous heating dissipation effect in Natural convection flows, shows that the heat transfer rates are reduced by an increase in the dissipation parameter. However the interaction of the radiation with mass transfer of hydromagnetic dynamics dissipative fluid in the presence of heat source has received little attention hence present study is attempted. [4] solved the problem of MHD flow with wavy porous boundary and the influences of heat source, suction and waviness of the boundaries of the flow were numerically analysed. The K-e' model is more reliable and likely to provide a superior performance for flow boundary layers under strong adverse pressure gradients. This was confirmed by comparing their findings with the experimental results. The research work cited above is the motivation of my current work. This 


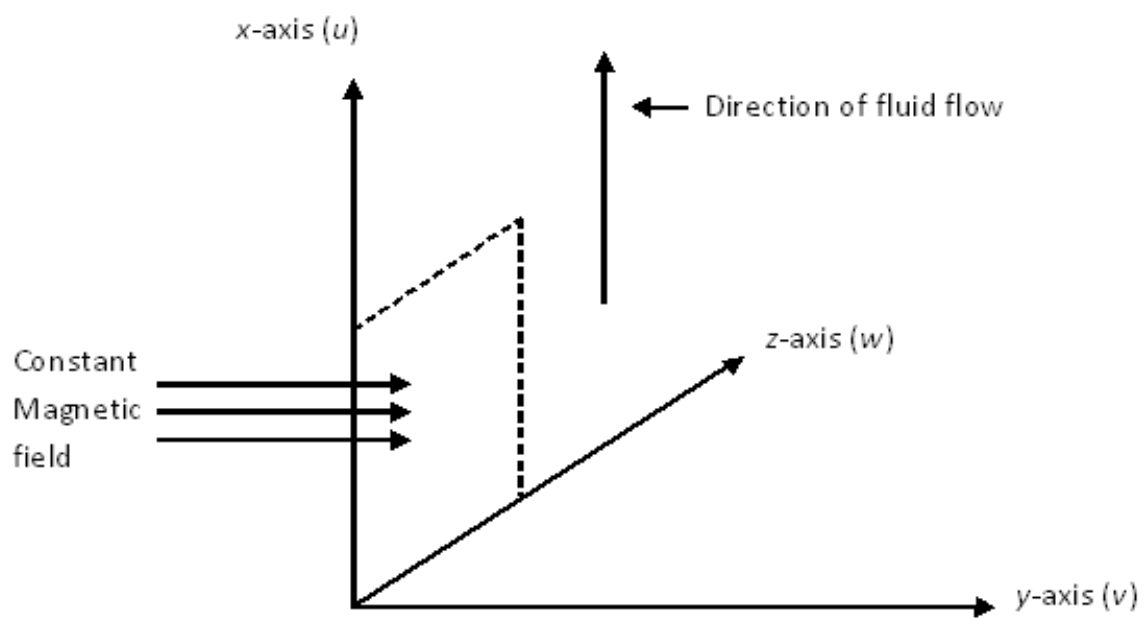

Figure 1: Flow Configuration

study considers heat and mass transfer for free convection fluid past porous finite parallel plates.

\section{Methodology}

\subsection{Mathematical Formulations}

Taking $x$ and $y$ as the coordinates parallel and normal to a flat plate respectively and $u, v$ and $w$ are taken as the velocities in the $x, y, z$ direction respectively. Since the plate is finite in length and for a two dimensional free convective fluid flow, the physical variables are functions of $x, y$ and $t$.

The equation of momentum along the $\mathrm{x}$-axis is

$$
\begin{aligned}
{\left[\frac{\partial u}{\partial t}+u \frac{\partial u}{\partial x}-V_{0} \frac{\partial u}{\partial y}\right]=v } & {\left[\frac{\partial^{2} u}{\partial x^{2}}+\frac{\partial^{2} u}{\partial y^{2}}\right] } \\
& +\beta g\left(T-T_{\infty}\right)+\beta^{*} g\left(C-C_{\infty}\right)-\frac{\sigma \mu_{e}^{2} H_{y}^{2} u}{\rho} .
\end{aligned}
$$


The equation of momentum along the $\mathrm{y}-$ axis is

$$
\left[\frac{\partial w}{\partial t}+u \frac{\partial w}{\partial x}-V_{0} \frac{\partial w}{\partial y}\right]=v\left[\frac{\partial^{2} w}{\partial x^{2}}+\frac{\partial^{2} w}{\partial y^{2}}\right]-\frac{\sigma \mu_{e}^{2} H_{y}^{2} w}{\rho}
$$

The equation of energy is

$$
\begin{aligned}
{\left[\frac{\partial T}{\partial t}+u \frac{\partial T}{\partial x}\right.} & \left.-V_{0} \frac{\partial T}{\partial y}\right] \\
& =\frac{1}{\rho C_{p}}\left\{k\left[\frac{\partial^{2} T}{\partial x^{2}}+\frac{\partial^{2} T}{\partial y^{2}}\right]+Q+\mu\left[\left(\frac{\partial u}{\partial y}\right)^{2}+\left(\frac{\partial w}{\partial y}\right)^{2}\right]\right\}
\end{aligned}
$$

The equation of concentration is

$$
\frac{\partial c}{\partial t}+u \frac{\partial c}{\partial x}-V_{0} \frac{\partial c}{\partial y}=D\left[\frac{\partial^{2} c}{\partial x^{2}}+\frac{\partial^{2} c}{\partial y^{2}}\right]
$$

Final set of non-dimensional governing equations

$$
\begin{aligned}
\frac{\partial u}{\partial t}+u \frac{\partial u}{\partial x}-V_{0} \frac{\partial u}{\partial y}= & {\left[\frac{\partial^{2} u}{\partial x^{2}}+\frac{\partial^{2} u}{\partial y^{2}}\right]+G r \theta+G c C-M^{2} u } \\
\frac{\partial w}{\partial t}+u \frac{\partial w}{\partial x}-V_{0} \frac{\partial w}{\partial y}= & {\left[\frac{\partial^{2} w}{\partial x^{2}}+\frac{\partial^{2} w}{\partial y^{2}}\right]-M^{2} w } \\
\frac{\partial \theta}{\partial t}+u \frac{\partial \theta}{\partial x}-V_{0} \frac{\partial \theta}{\partial y}= & \frac{1}{\operatorname{Pr}}\left[\frac{\partial^{2} \theta}{\partial y^{2}}+\frac{\partial^{2} \theta}{\partial x^{2}}\right]+\frac{\delta}{\operatorname{Pr} \theta} \\
& +E_{c}\left[\left(\frac{\partial u}{\partial y}\right)^{2}+\left(\frac{\partial w}{\partial y}\right)^{2}\right] \\
\frac{\partial C}{\partial t}+u \frac{\partial C}{\partial x}-V_{0} \frac{\partial C}{\partial y}= & \frac{1}{S c}\left(\frac{\partial^{2} C}{\partial x^{2}}+\frac{\partial^{2} C}{\partial y^{2}}\right)
\end{aligned}
$$

Initial conditions are

$$
\begin{gathered}
\text { At } y=0, u_{(0, i)}^{0}=1, w_{(0, i)}^{0}=0, \theta_{(0, i)}^{0}=1, c_{(0, i)}^{0}=1 . \\
y>0, u_{(k, i)}^{0}=0, w_{(k, i)}^{0}=0, \theta^{0}=1, c_{(k, i)}^{0}=0 .
\end{gathered}
$$

$k>0$ and all $i$ the boundary conditions takes the form

At $y=0, u_{(0, i)}^{n}=1, w_{(0, i)}^{n}=0, \theta_{(0, i)}^{0}=1, c_{(0, i)}^{n}=1$

$$
x=0, u_{(k, 0)}^{n}=1, w_{(k, 0)}^{n}=0, \theta_{(k, i)}^{n}=1, c_{(k, i)}^{n}=0
$$




$$
\begin{aligned}
& u_{(k, i)}^{n+1}=u_{(k, i)}^{n}+\left[\begin{array}{l}
-u_{(k, i)}^{n}\left[\frac{u_{(k, i+1)}^{n}-u_{(k, i-1)}^{n}}{2 \Delta x}\right]+V_{0}^{n}\left[\frac{u_{(k+1, i)}^{n}-u_{(k-1, i)}^{n}}{2 \Delta y}\right] \\
+\left[\frac{u_{(k+1, i)}^{n}-2 u_{(k, i)}^{n}+u_{(k-i, i)}^{n}}{(\Delta y)^{2}}\right]+\left[\frac{u_{(k, i+1)}^{n}-2 u_{(k, i)}^{n}+u_{(k, i-1)}^{n}}{(\Delta x)^{2}}\right] \\
+G_{r} \theta_{(k, i)}^{n}+G_{c} C_{(k, i)}^{n}-M^{2} u_{(k, i)}^{n}
\end{array}\right] \Delta t \\
& w_{(k, i)}^{n+1}=w_{(k, i)}^{n}+\left[\begin{array}{l}
-u_{(k, i)}^{n}\left[\frac{w_{(k, i+1)}^{n}-w_{(k, i-1)}^{n}}{2 \Delta x}\right]+V_{0}^{n}\left[\frac{w_{(k+1, i)}^{n}-w_{(k-1, i)}^{n}}{2 \Delta y}\right]+ \\
+\left[\frac{w_{(k+1, i)}^{n}-2 w_{(k, i)}^{n}+w_{(k-1, i)}^{n}}{(\Delta y)^{2}}\right]+\left[\frac{w_{(k, i+1)}^{n}-2 w_{(k, i)}^{n}+w_{(k, i-1)}^{n}}{(\Delta x)^{2}}\right] \\
-M^{2} w_{(k, i)}^{n}
\end{array}\right] \Delta t \\
& \theta_{(k, i)}^{n+1}=\theta_{(k, i)}^{n}+\left[\begin{array}{l}
-u_{(k, i)}^{n}\left[\frac{\theta_{(k, i+1)}^{n}-\theta_{(k, i-1)}^{n}}{2 \Delta x}\right]+V_{0}^{n}\left[\frac{\left.\theta_{(k+1, i)}^{n}-\theta_{(k-1, i)}^{n}\right]+}{2 \Delta y}\right]^{\frac{1}{p_{r}}}\left[\frac{\theta_{(k, i+1)}^{n}-2 \theta_{(k, i)}^{n}+\theta_{(k, i-1)}^{n}}{(\Delta y)^{2}}\right]+\frac{1}{p_{r}}\left[\frac{\theta_{(k+1, i)}^{n}-2 \theta_{(k, i)}^{n}+\theta_{(k-1, i)}^{n}}{(\Delta x)^{2}}\right] \\
+\frac{\delta}{p_{r}} \theta_{(k, i)}^{n}+E_{c}\left[\frac{u_{(k, i+1)}^{n}-u_{(k, i-1)}^{n}}{2 \Delta y}\right]^{2}+E_{c}\left[\frac{w_{(k, i+1)}^{n}-w_{(k, i-1)}^{n}}{2 \Delta y}\right]^{2}
\end{array}\right] \\
& C_{(k, i)}^{n+1}=C_{(k, i)}^{n}+\left[\begin{array}{l}
-u_{(k, i)}^{n}\left[\frac{C_{(k, i+1)}^{n}-C_{(k, i-1)}^{n}}{2 \Delta x}\right]+V_{0}^{n}\left[\frac{C_{(k+1, i)}^{n}-C_{(k-1, i)}^{n}}{2 \Delta y}\right]+ \\
\frac{1}{S c}\left[\frac{C_{(k+1, i)}^{n}-2 C_{(k, i)}^{n}+C_{(k-1, i)}^{n}}{(\Delta y)^{2}}\right]+\frac{1}{S c}\left[\frac{C_{(k, i+1)}^{n}-2 C_{(k, i)}^{n}+C_{(k, i-1)}^{n}}{(\Delta x)^{2}}\right]
\end{array}\right]
\end{aligned}
$$

As the $\mathrm{x}$-axis is long the finite vertical plate then $x$ varies from 0 to infinity. The computations are performed using small values $\triangle x$ and $\triangle y$ where in the research we set $\triangle x=\triangle y=1$. Fixing $y=3.1$ form which $k=31$ as corresponding to $y=\infty$ therefore set. $V_{(31, i)}^{n}=U_{(31, i)}^{n}=W_{(31, i)}^{n}=C_{(31, i)}^{n}=\theta_{(31, i)}^{n}=0$ because $v, u, w, c$ and $\theta$ tends to zero around $y=3.1$. The boundary condition takes the form.

Initial conditions are:

$$
\begin{gathered}
\text { At } y=0, u_{(0, i)}^{0}=1, w_{(0, i)}^{0}=0, \theta_{(0, i)}^{0}=1, c_{(0, i)}^{0}=1 \\
y>0, u_{(k, i)}^{0}=0, w_{(k, i)}^{0}=0, \theta^{0}=1, c_{(k, i)}^{0}=0
\end{gathered}
$$

$k>0$ and all $i$ the boundary conditions takes the form

$$
\begin{gathered}
\text { At } y=0, u_{(0, i)}^{n}=1, w_{(0, i)}^{n}=0, \theta_{(0, i)}^{0}=1, c_{(0, i)}^{n}=1 \\
x=0, u_{(k, 0)}^{n}=1, w_{(k, 0)}^{n}=0, \theta_{(k, 0)}^{n}=0, c_{(k, 0)}^{n}=0
\end{gathered}
$$

In the computation the prandtl number is taken as 0.71 which corresponds to air, magnetic parameter $M^{2}=5.0$ signifying a strong magnetic field and Grashof number, $G r$ is varying from $0,-0.2,-0.4,-0.6,-0.8,-1$ and corresponding to convective heating of the plate. To ensure stability and convergence 


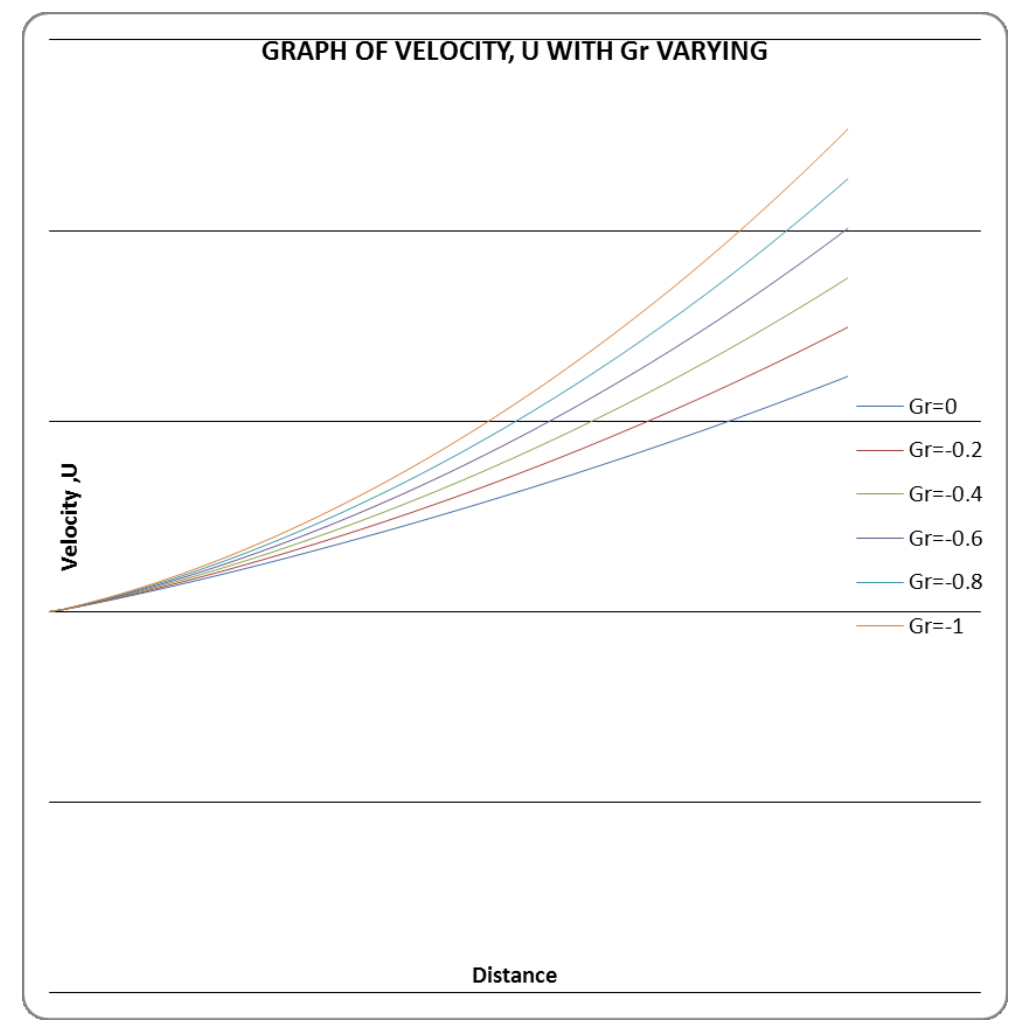

Figure 2: Primary velocity profile (u-graph)

of the finite difference method, the program is run using smaller values of $\triangle y$ and $\triangle x$ such as $0.0001,0.00005,0.000015$.

\section{Results}

\subsection{Primary Velocity Profiles}

From Figure 2, it is observed that as the Grashof parameter decreases primary velocity profiles increases and as the distance from the plates increase, the curve exhibits an increase in primary velocity profile from curves $\mathrm{Gr}=0, \mathrm{Gr}=-0.2$, $\mathrm{Gr}=-0.4, \mathrm{Gr}=-0.6, \mathrm{Gr}=-0.8, \mathrm{Gr}=-1$ respectively (heating of the plate by free convection current). 


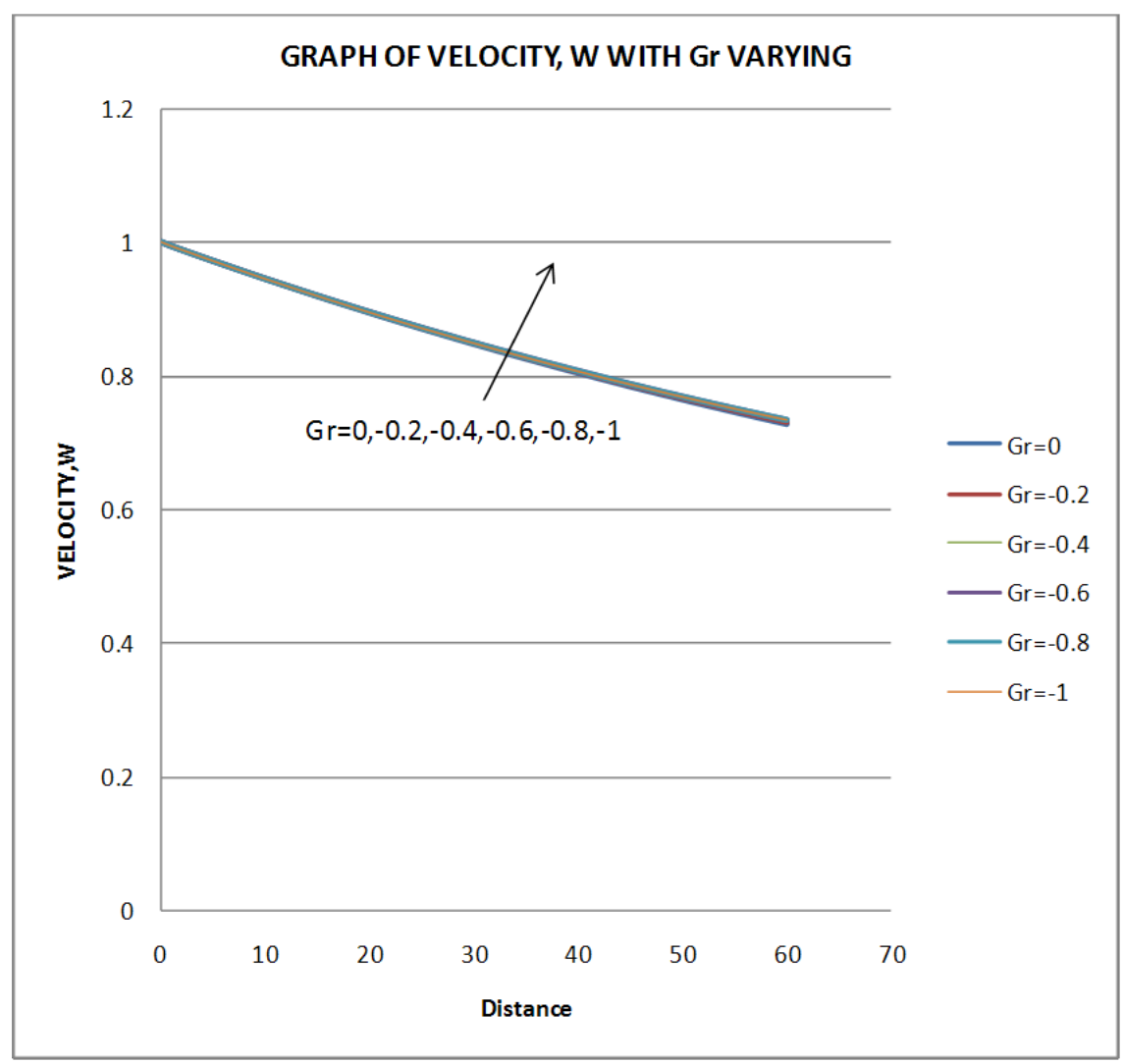

Figure 3: Secondary velocity profile (w-graph)

\subsection{Secondary Velocity Profiles}

From Figure 3, it is observed that as the Grashof parameter decreases from 0 to -1 the secondary velocities profiles increases but as distance from the plate increases the curve exhibits a decrease in the velocity profile from the curves $\mathrm{Gr}=0, \mathrm{Gr}=-0.2, \mathrm{Gr}=-0.4, \mathrm{Gr}=-0.6, \mathrm{Gr}=-0.8, \mathrm{Gr}=-1$ respectively (heating of the plate by free convection current).

\subsection{Temperatures Profile}

From Figure 4, it is observed that as the Grashof parameter decreases from 0 to -1 the temperature profiles increases, also as the distance from the plate increases the curve exhibits an increase in temperature profiles from curves 


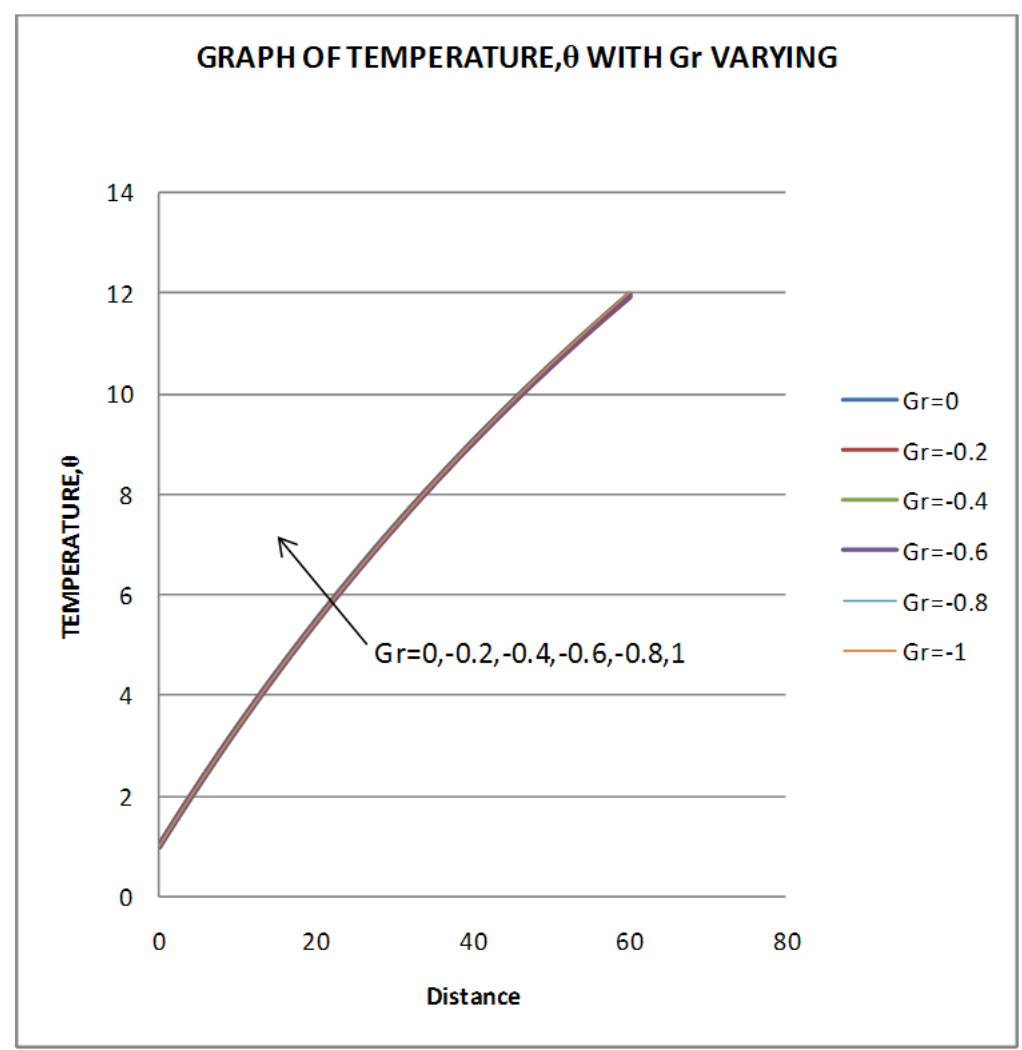

Figure 4: Temperature profile ( $\vartheta$-graph)

$\mathrm{Gr}=0, \mathrm{Gr}=-0.2, \mathrm{Gr}=-0.4, \mathrm{Gr}=-0.6, \mathrm{Gr}=-0.8, \mathrm{Gr}=-1$ respectively (heating of the plate by free convection current).

\subsection{Concentration Profile}

From the Figure 5, it is observed that as the Grashof parameter decreases from 0 to -1 the concentration profiles increases but as distance from the plate increases the concentration profile decreases exponentially as exhibited by the curves $\mathrm{Gr}=0, \mathrm{Gr}=-0.2, \mathrm{Gr}=-0.4, \mathrm{Gr}=-0.6, \mathrm{Gr}=-0.8, \mathrm{Gr}=-1$ respectively (heating of the plate by free convection current). 


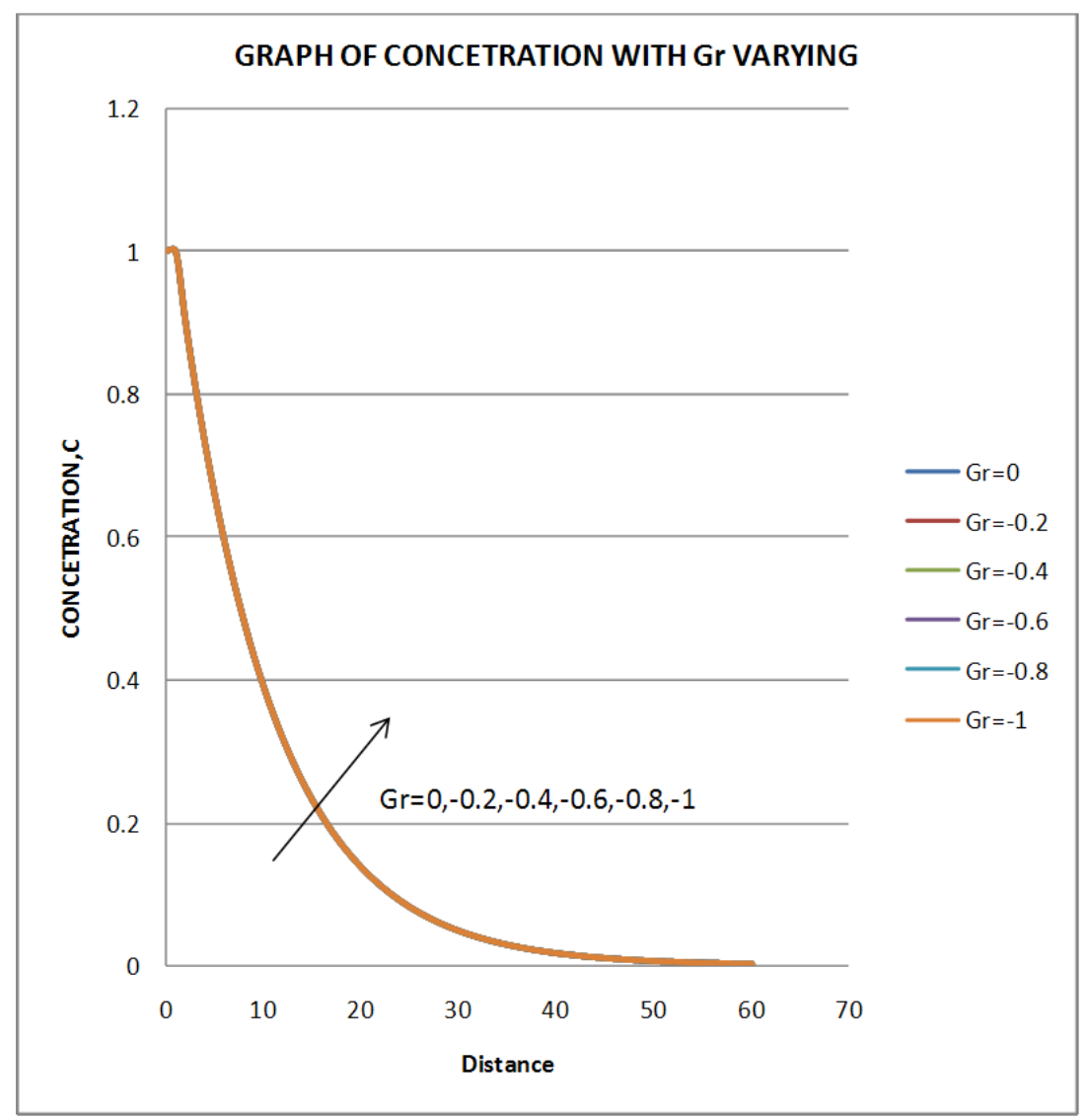

Figure 5: Concentration profile (c-graph)

\section{Conclusion}

An analysis of the buoyancy effects of thermal and mass diffusion on velocities, temperature and concentration profiles on MHD natural convection past a finite vertical plate has been carried out. In all the cases considered, the velocity was resolved into two components and the work restricted to laminar boundary layer.

In the study the results obtained for $G r<0$ when the temperature of the plate is less than that of the fluid in the free stream region, which implies that the heat was being transferred from the fluid to the plate which refers to the heating of the plate by free convection currents.

The results obtained for various values of Grashof parameter were presented 
graphically and in table form. It can be concluded that a decrease in Grashof parameter leads to an increase in primary, secondary, temperature and concentration profiles.

\section{References}

[1] Chamkha, A. K. (2004). Unsteady mhd convection heat and mass transfer past a semi-infinite vertical permeable moving plate with heat absorption. International Journal of Engineering Science, 42:217-230.

[2] El-Amin, M. (2001). Magnetohydrodynamic free convection and mass transfer flow in micro polar fluid with constant suction. Journal of Magnetism and magnetic Materials, 234:567-574.

[3] Hossain and Mondal (1985). Mass transfer effects on the unsteady hydromagnetic free convection flow past an accelerated porous plate. Journal of Physics D Applied Physics, 18(7):32, 89, 133.

[4] Prasada (1985). Mhd flow in a channel with wavy porous boundary. Proceedings of the National Academy of Sciences of the United States of America, $51 \mathrm{~A}(2): 391-406$.

[5] Revankar (1983). Natural convection effects on mhd flow pat an impulsively started permeable plate. Indian Journal of Pure and Applied Mathematics, 14:530-539.

[6] Singh, V. (1989). Unsteady mhd flow of an incompressible dusty-viscous conducting fluid through an open rectangular channel with permeable bottom. Indian Journal of Theoretical Physics, 37:19-36.

[7] Yan, W. and Lin, T. F. (1987). Natural convection heat transfer in vertical open channel flows with discrete heating. International Communications in Heat and Mass Transfer, 14(2):187-200. 